Supporting Information

\title{
Hydrogen Evolving Activity of Dithiolene-Based Metal-Organic Frameworks with Mixed Cobalt and Iron Centers
}

\author{
Keying Chen ${ }^{*}$, Courtney A. Downes ${ }^{*}$, Jason D. Goodpaster ${ }^{\S}$, and Smaranda C. Marinescu **
}

Department of Chemistry, University of Southern California, Los Angeles, California 90089, United States.

${ }^{\S}$ Department of Chemistry, University of Minnesota - Twin Cities, Minneapolis, Minnesota 55455, United States

*Email: smarines@usc.edu.

\section{General}

All manipulations of air and moisture sensitive materials were conducted under a nitrogen atmosphere in a Vacuum Atmospheres Glovebox or on a dual manifold Schlenk line. The glassware was oven-dried prior to use. Water was deionized with the Millipore Synergy system $(18.2 \mathrm{M} \Omega \cdot \mathrm{cm}$ resistivity). All the solvents used were degassed under vacuum and refilled with nitrogen $(10 \times)$. Triphenylene-2,3,6,7,10,11-hexathiol ligand (THT) was synthesized according to literature procedures. ${ }^{1}$ All other chemical reagents were purchased from commercial vendors and used without further purification.

\section{Synthesis of FeTHT}

The synthesis of FeTHT was previously reported by our laboratory. ${ }^{2}$ A $120 \mathrm{~mL}$ jar was charged with a solution of $\mathrm{FeCl}_{2} \cdot 4 \mathrm{H}_{2} \mathrm{O}(40 \mathrm{mg}, 0.20 \mathrm{mmol})$ in water $(40 \mathrm{~mL})$. Separately, a suspension of THT (2.5 mg, $0.006 \mathrm{mmol})$ in N-methyl-2-pyrrolidone (NMP) $(0.1 \mathrm{~mL})$ was diluted with ethyl acetate until the total volume of the suspension reached $5 \mathrm{~mL}$, sealed, and briefly sonicated to form a uniform suspension. Ethyl acetate $(35 \mathrm{~mL})$ was gently layered on top of the aqueous solution to create a liquid-liquid interface; the suspension of THT was then gently added to the ethyl acetate layer and the jar was sealed and allowed to stand still. A black film appeared at the liquid-liquid interface over 5 days, which was then collected as powder, solvent exchanged with water $(3 \times 20 \mathrm{~mL})$, methanol $(3 \times 20 \mathrm{~mL})$, and dried under vacuum.

\section{Synthesis of mixed Fe/Co THT}

A series of aqueous solutions with different molar ratios of $\mathrm{FeCl}_{2} \cdot 4 \mathrm{H}_{2} \mathrm{O}$ and $\mathrm{CoCl}_{2} \cdot 6 \mathrm{H}_{2} \mathrm{O}$ were prepared and added to a $120 \mathrm{~mL}$ jar. The total metal amount was controlled to be $0.20 \mathrm{mmol}$ for each jar. Separately, a suspension of THT $(2.5 \mathrm{mg}, 0.006 \mathrm{mmol})$ in N-methyl-2-pyrrolidone (NMP) $(0.1 \mathrm{~mL})$ was diluted with ethyl acetate until the total volume of the suspension reached 5 $\mathrm{mL}$, sealed, and briefly sonicated to form a uniform suspension. Ethyl acetate $(35 \mathrm{~mL})$ was gently layered on top of the aqueous solution to create a liquid-liquid interface; the suspension of 
THT was then gently added to the ethyl acetate layer and the jar was sealed and allowed to stand still. A black film appeared at the liquid-liquid interface over 5 days, which was then collected as powder, solvent exchanged with water $(3 \times 20 \mathrm{~mL})$, methanol $(3 \times 20 \mathrm{~mL})$, and dried under vacuum.

\section{Deposition of MOFs for Electrochemistry Study}

The THT-based MOFs were deposited as an ink mixture. To generate the ink mixture, $2 \mathrm{mg}$ of as-prepared MOF powder was mixed with $0.5 \mathrm{mg}$ of carbon black (Vulcan XC-72R), $20 \mu \mathrm{L}$ of Nafion solution $(0.5 \mathrm{wt} \%), 45 \mu \mathrm{L}$ of water, and $135 \mu \mathrm{L}$ of ethanol, followed by sonication for 30 mins to form a uniformly dispersed suspension. A desired amount of this suspension was then drop casted onto a freshly polished glassy carbon electrode (GCE) or a rotating disk electrode (RDE, glassy carbon insert) using a micro syringe, and dried in a nitrogen atmosphere at room temperature.

\section{Electrochemical Methods}

Electrochemistry experiments were carried out using a VersaSTAT 3 potentiostat in a three-electrode configuration electrochemical cell under an inert atmosphere. A glassy carbon electrode (GCE, $0.07065 \mathrm{~cm}^{2}$ surface area, used for CV studies) or a rotating disk electrode (RDE, glassy carbon insert, $0.196 \mathrm{~cm}^{2}$ surface area, used for obtaining the steady-state polarization curves) was used as the working electrode. GCE and RDE were polished with 0.05 $\mu \mathrm{m} \mathrm{Al}_{2} \mathrm{O}_{3}$ polish powder and sonicated in water prior to use. A graphite rod, purchased from Graphite Machining, Inc. (Grade NAC-500 Purified, $<10 \mathrm{ppm}$ ash level), was used as the counter electrode. The reference electrode, placed in a separate compartment and connected by a Vycor tip, was based on an aqueous $\mathrm{Ag} / \mathrm{AgCl} /$ saturated $3.5 \mathrm{M} \mathrm{KCl}$ electrode. The reference electrode in aqueous media was calibrated externally relative to ferrocenecarboxylic acid $(\mathrm{Fc}-\mathrm{COOH})$ at $\mathrm{pH} 7.0$, with the $\mathrm{Fe}^{3+/ 2+}$ couple at $0.28 \mathrm{~V}$ vs. $\mathrm{Ag} / \mathrm{AgCl}$. All potentials reported in this paper were converted to the reversible hydrogen electrode (RHE) by adding a value of $(0.205+0.059 \times \mathrm{pH}) \mathrm{V}$, or to the normal hydrogen electrode (NHE) by adding a value of 0.205 $\mathrm{V}$ (for $\mathrm{pH} 10 \mathrm{CV}$ studies). The aqueous solutions used in the electrochemical experiments were prepared as follows. For the $\mathrm{pH} 1.3$ solution, $0.534 \mathrm{~mL}$ of $18.7 \mathrm{M} \mathrm{H}_{2} \mathrm{SO}_{4}$ was added to $200 \mathrm{~mL}$ $0.1 \mathrm{M} \mathrm{NaClO}$. For the $\mathrm{pH} 10.0$ solution, $\mathrm{NaHCO}_{3}(0.678 \mathrm{~g})$ and $\mathrm{Na}_{2} \mathrm{CO}_{3}$ (1.264 g) were dissolved in $200 \mathrm{~mL} 0.1 \mathrm{M} \mathrm{NaClO}_{4}$. The $\mathrm{pH}$ of the solutions was measured with a benchtop Mettler Toledo $\mathrm{pH}$ meter. Prior to each electrochemical experiment, the electrolyte solution was purged with nitrogen thoroughly.

Controlled potential electrolysis (CPE) measurements to determine Faradaic efficiency were conducted in a sealed two-chambered $\mathrm{H}$-cell where the first chamber held the working and reference electrodes in $40 \mathrm{~mL}$ of $\mathrm{pH} 1.3$ aqueous solution and the second chamber held the counter electrode in $20 \mathrm{~mL}$ of $\mathrm{pH} 1.3$ aqueous solution. The two chambers were both under $\mathrm{N}_{2}$ and separated by a fine porosity glass frit. CPE experiments were performed with a glassy carbon plate electrode $(6 \mathrm{~cm} \times 1 \mathrm{~cm} \times 0.3 \mathrm{~cm}$; Tokai Carbon USA) as the working electrode and a graphite rod as the counter electrode. The reference electrode was a $\mathrm{Ag} / \mathrm{AgCl} / \mathrm{saturated} 3.5 \mathrm{M}$ $\mathrm{KCl}$ (aq.) electrode separated from the solution by a Vycor tip. Using a gas-tight syringe, $2 \mathrm{~mL}$ of gas was withdrawn from the headspace of the H-cell and injected into a gas chromatography 
instrument (Shimadzu GC-2010-Plus) equipped with a BID detector and a Restek ShinCarbon ST Micropacked column. To determine the Faradaic efficiency, the theoretical $\mathrm{H}_{2}$ amount based on total charge flowed is compared with the GC-detected $\mathrm{H}_{2}$ produced from controlled-potential electrolysis.

Electrochemical impedance spectroscopy (EIS) measurements were carried out at different overpotentials in the frequency range of $100 \mathrm{kHz}-0.1 \mathrm{~Hz}$ with $10 \mathrm{mV}$ sinusoidal perturbations. Experimental EIS data were analyzed and fitted with the ZSimpWin software.

The obtained polarization curves were corrected for $i R$ loss according to the following equation: $E_{\text {corr }}=E_{\text {mea }}-i R_{\mathrm{s}}$

Where $E_{\text {corr }}$ is the $i R$-corrected potential, $E_{\text {mea }}$ is the experimentally measured potential, and $R_{\mathrm{s}}$ is the solution resistance extracted from the fitted EIS data.

\section{Physical Characterization Methods}

X-ray photoelectron spectroscopy (XPS) data were collected using a Kratos AXIS Ultra instrument. The monochromatic X-ray source was the $\mathrm{Al} \mathrm{K \alpha}$ line at $1486.7 \mathrm{eV}$, and the hybrid lens and slot mode were used. Low resolution survey spectra were acquired between binding energies of 1-1200 eV. Higher resolution detailed scans, with a resolution of $0.1 \mathrm{eV}$, were collected on individual XPS regions of interest. The sample chamber was maintained at $<$ $9 \times 10^{-9}$ Torr. The XPS data were analyzed using the CasaXPS software.

High resolution synchrotron powder X-ray diffraction data was collected using the 11-BM beamline mail-in program at the Advanced Photon Source (APS), Argonne National Laboratory, with an average wavelength of $0.412750 \AA$. Discrete detectors covering an angular range from 0.5 to $30^{\circ} 2 \theta$ are scanned over a $34^{\circ} 2 \theta$ range, with data points collected every $0.001^{\circ} 2 \theta$ and scan speed of $0.01 \%$.

In-house powder X-ray diffraction (PXRD) studies were performed on a Rigaku Ultima IV $\mathrm{X}$-Ray diffractometer in reflectance parallel beam/parallel slit alignment geometry. The measurement employed $\mathrm{Cu} \mathrm{K} \alpha$ line focused radiation at $1760 \mathrm{~W}(40 \mathrm{kV}, 44 \mathrm{~mA})$ power and a $\mathrm{Ge}$ crystal detector fitted with a $2 \mathrm{~mm}$ radiation entrance slit. Samples were mounted on zero-background sample holders and were observed using a $0.08^{\circ} 2 \theta$ step scan from $2.0-40.0^{\circ}$ with an scan rate of $1^{\circ}$ per minute.

Inductively coupled plasma optical emission spectroscopy (ICP-OES) measurements were performed using a Thermo Scientific iCAP 7000 ICP-OES. After electrochemical tests, the composite was removed from the electrode surface, digested in concentrated nitric acid, and tested by ICP-OES to determine the concentration of the Co and Fe in the catalyst. The post-CPE electrolyte solution samples were prepared by adding certain amount of nitric acid to ensure the consistency of the background.

Scanning electron microscopy (SEM) and energy-dispersive X-ray spectroscopy (EDX) were performed on an FEI Nova NanoSEM 450 scanning electron microscope using an accelerating voltage of $15 \mathrm{kV}$. 
Table S1. Metal content in mixed metal THT-based MOFs

\begin{tabular}{|c|c|c|c|c|c|c|}
\hline \multirow{2}{*}{ Material } & $\begin{array}{c}\text { Feeding } \\
\text { Fe:Co } \\
\text { molar ratio }\end{array}$ & \multicolumn{2}{|c|}{ Product Fe:Co Molar Ratio } & \multirow{2}{*}{$\begin{array}{c}\text { Average Fe:Co } \\
\text { Ratio }\end{array}$} & $\begin{array}{c}\text { Average } \\
\text { Fe\% }\end{array}$ \\
\hline $\mathbf{1}$ & 1000 & 3.21 & 3.14 & 3.22 & $3.19 \pm 0.04$ & $76.1 \%$ \\
\hline $\mathbf{2}$ & 100.0 & $9.52 \times 10^{-1}$ & $7.58 \times 10^{-1}$ & $8.55 \times 10^{-1}$ & $(8.54 \pm 0.97) \times 10^{-1}$ & $46.1 \%$ \\
\hline $\mathbf{3}$ & 1.00 & $5.24 \times 10^{-2}$ & $6.13 \times 10^{-2}$ & $5.46 \times 10^{-2}$ & $(5.61 \pm 0.47) \times 10^{-2}$ & $5.29 \%$ \\
\hline
\end{tabular}
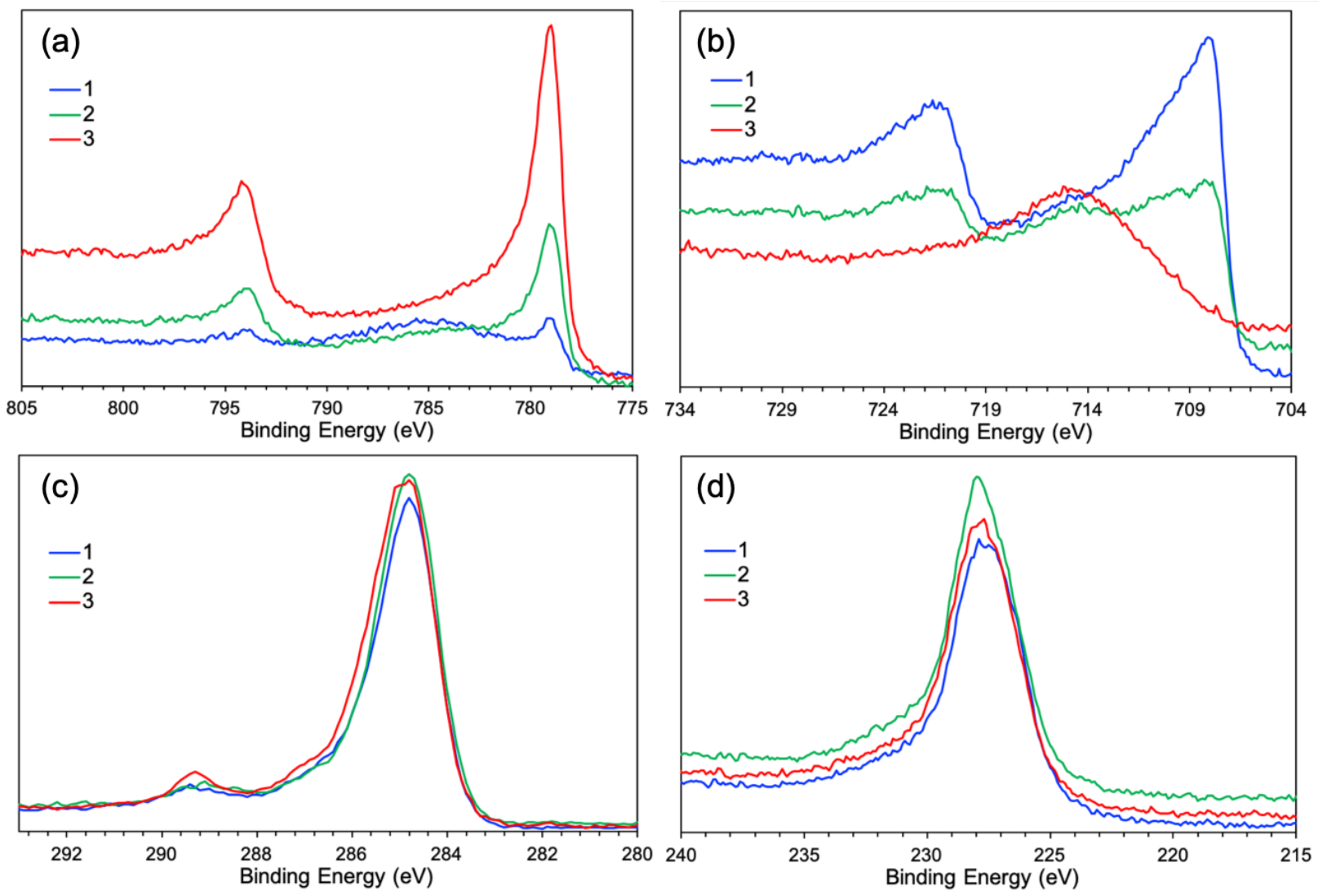

Figure S1. High resolution XPS spectra of 1, 2, and 3: (a) Co 2p region; (b) Fe 2p region; (c) C 1s region; (d) S 2s region. 


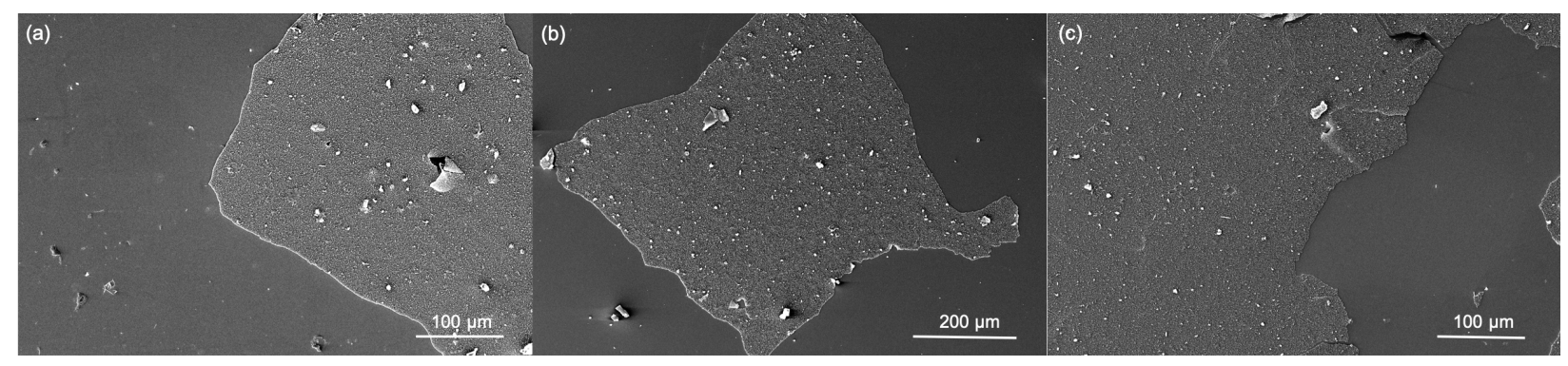

Figure S2. Top-down SEM images of (a) 1, (b) 2, and (c) 3.

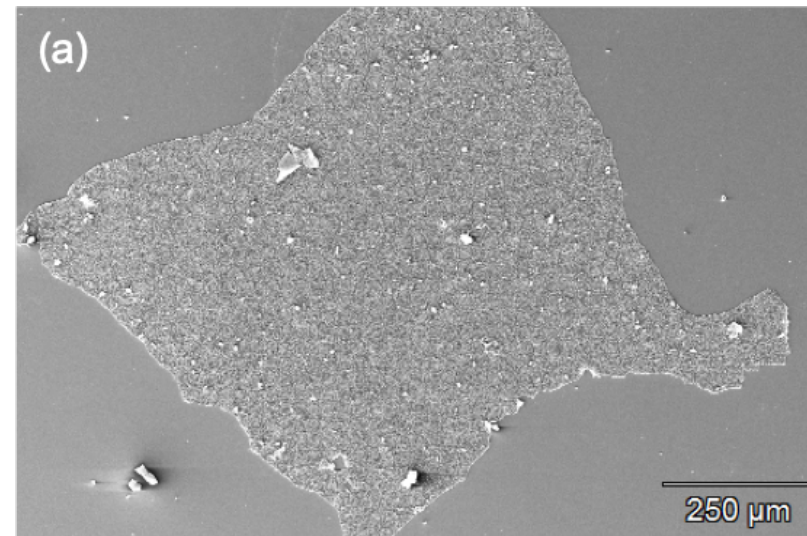

(b)

(c)

(d)

Figure S3. (a) Top-down SEM image of 2. EDX spectra of 2 revealing the presence and homogeneous distribution of (b) S, (c) Co, and (d) Fe. 

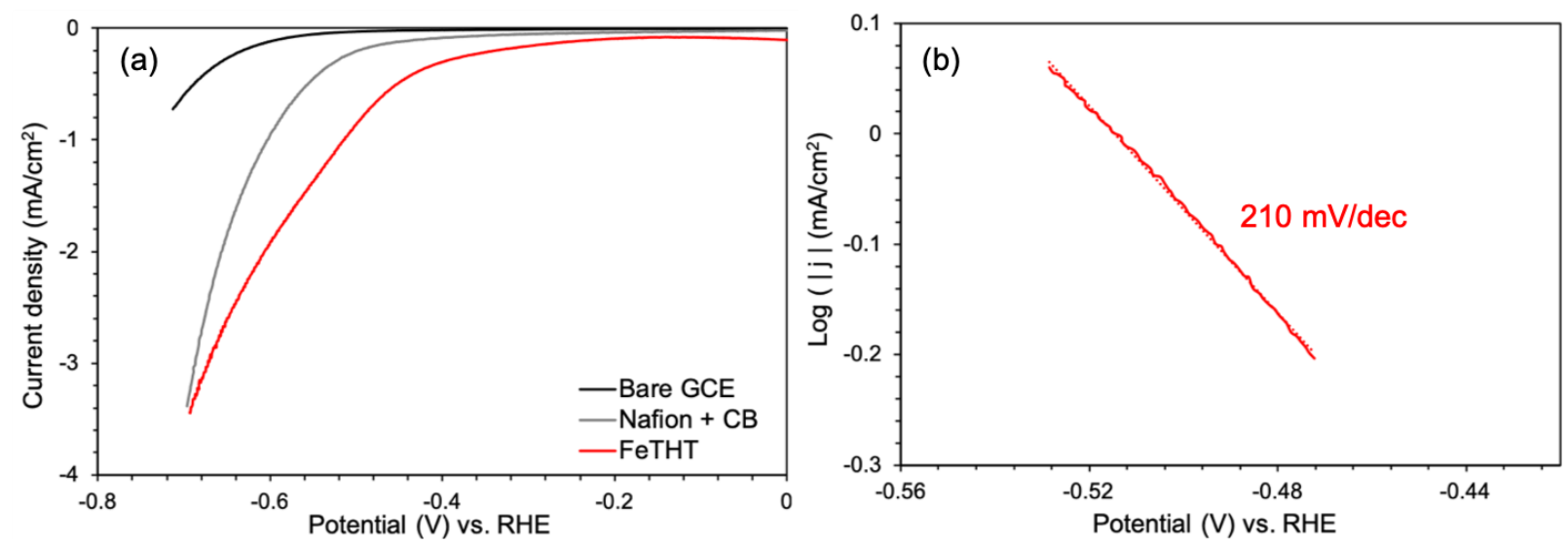

Figure S4. (a) Polarization curves of FeTHT (red), bare glassy carbon electrode (black), and components of the ink (grey). (b) Tafel plot obtained from polarization curve. Scan rate: $5 \mathrm{mV} / \mathrm{s}$, rotation rate of RDE: $2000 \mathrm{rpm}$, loading of catalyst: $2.85 \times 10^{-7} \mathrm{~mol}_{\mathrm{Fe}} / \mathrm{cm}^{2}$. All measurements were performed in $\mathrm{N}_{2}$-saturated $\mathrm{pH} 1.3$ aqueous solutions.

(a)
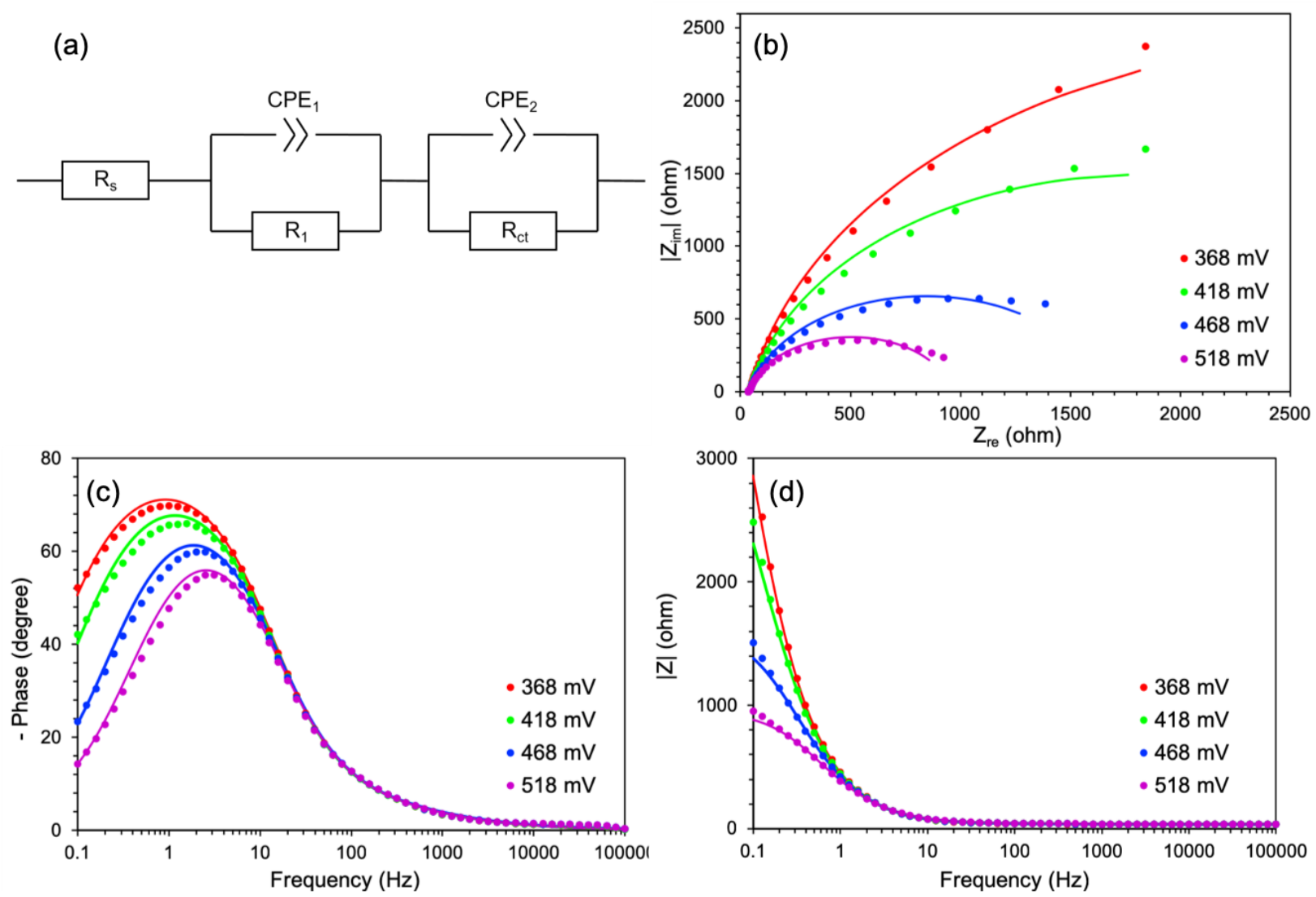

Figure S5. (a) The two-time constant serial model (2TS) employed to fit the EIS response of FeTHT. (b) Nyquist plots (markers) of FeTHT at various overpotentials with respective fits (solid lines). (c) and (d): Bode plots (markers) of FeTHT at various overpotentials with respective fits (solid lines). All measurements were performed in $\mathrm{N}_{2}$-saturated $\mathrm{pH} 1.3$ aqueous solutions. 
Table S2. $\mathrm{R}_{\mathrm{ct}}$ values of FeTHT derived from fitting EIS data to the 2TS model

\begin{tabular}{ccccc}
\hline $\boldsymbol{\eta}(\mathbf{m V})$ & $\mathbf{3 6 8}$ & $\mathbf{4 1 8}$ & $\mathbf{4 6 8}$ & $\mathbf{5 1 8}$ \\
\hline $\mathrm{R}_{\mathrm{ct}}(\Omega)$ & 5677 & 3581 & 1605 & 922.7 \\
\hline
\end{tabular}

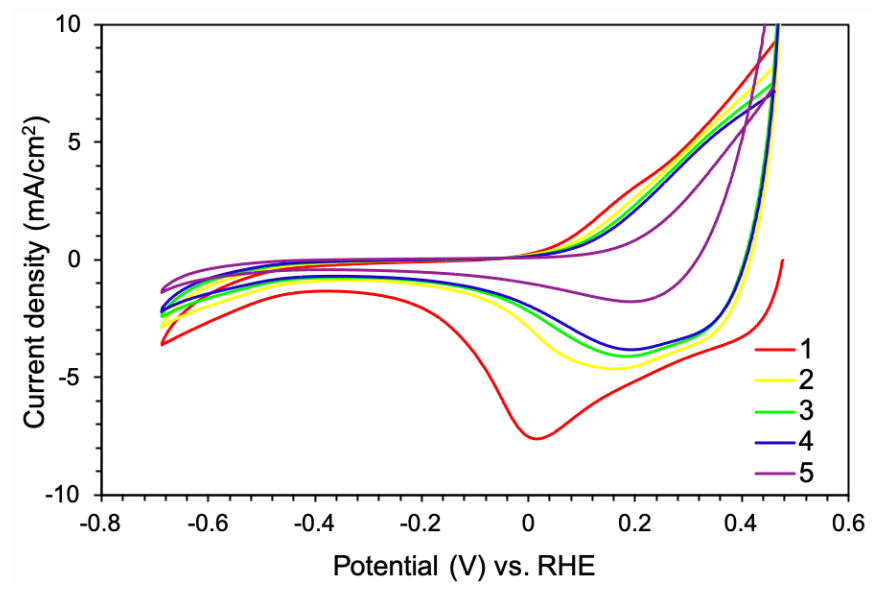

Figure S6. Consecutive CV scans of FeTHT in a $\mathrm{N}_{2}$-saturated $\mathrm{pH} 1.3$ aqueous solution, scan rate: $50 \mathrm{mV} / \mathrm{s}$.
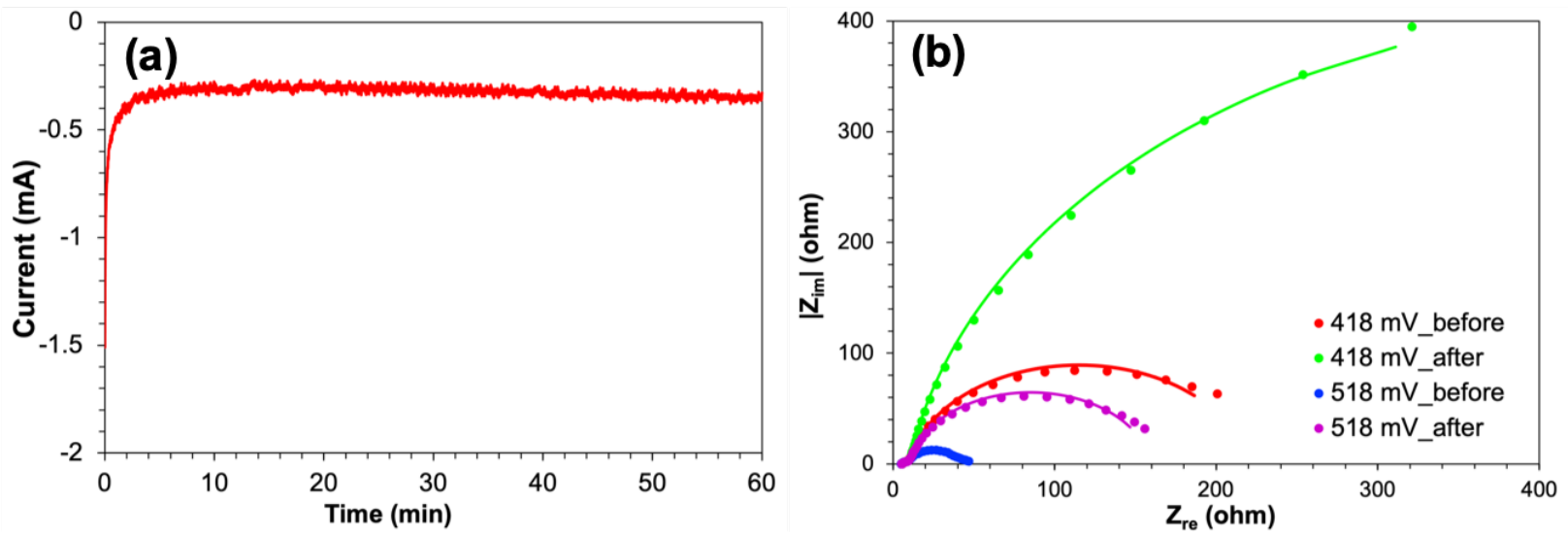

Figure S7. (a) $1 \mathrm{hr}$ CPE experiment of FeTHT at $-0.518 \mathrm{~V}$ vs. RHE, FE: $72.2 \pm 1.4 \%$. (b) Nyquist plots (marks) fitted to 2TS model (lines) showing EIS responses at different overpotentials before and after CPE. All measurements were performed in a $\mathrm{N}_{2}$-saturated $\mathrm{pH} 1.3$ aqueous electrolyte solution. 
Table S3. Characteristic values of FeTHT extracted from the EIS responses shown in Figure S7b.

\begin{tabular}{c|ccc|ccc}
\hline \multirow{2}{*}{$\begin{array}{c}\text { Potential } \\
(\mathbf{V} \text { vs. } \mathbf{R H E})\end{array}$} & \multicolumn{3}{|c|}{ Before CPE } & \multicolumn{3}{c}{ After CPE } \\
\cline { 2 - 7 } & $\mathbf{R}_{\mathbf{S}}(\boldsymbol{\Omega})$ & $\mathbf{R}_{\mathbf{c t}}(\boldsymbol{\Omega})$ & $\mathbf{C}_{\mathbf{d l}}(\mathbf{m F})$ & $\mathbf{R}_{\mathbf{S}}(\boldsymbol{\Omega})$ & $\mathbf{R}_{\mathbf{c t}}(\mathbf{\Omega})$ & $\mathbf{C}_{\mathbf{d l}}(\mathbf{m F})$ \\
\hline-0.418 & 5.48 & 210 & 1.65 & 5.84 & 940 & 1.64 \\
\hline-0.518 & 5.15 & 35.0 & 1.58 & 5.78 & 151 & 1.64 \\
\hline
\end{tabular}
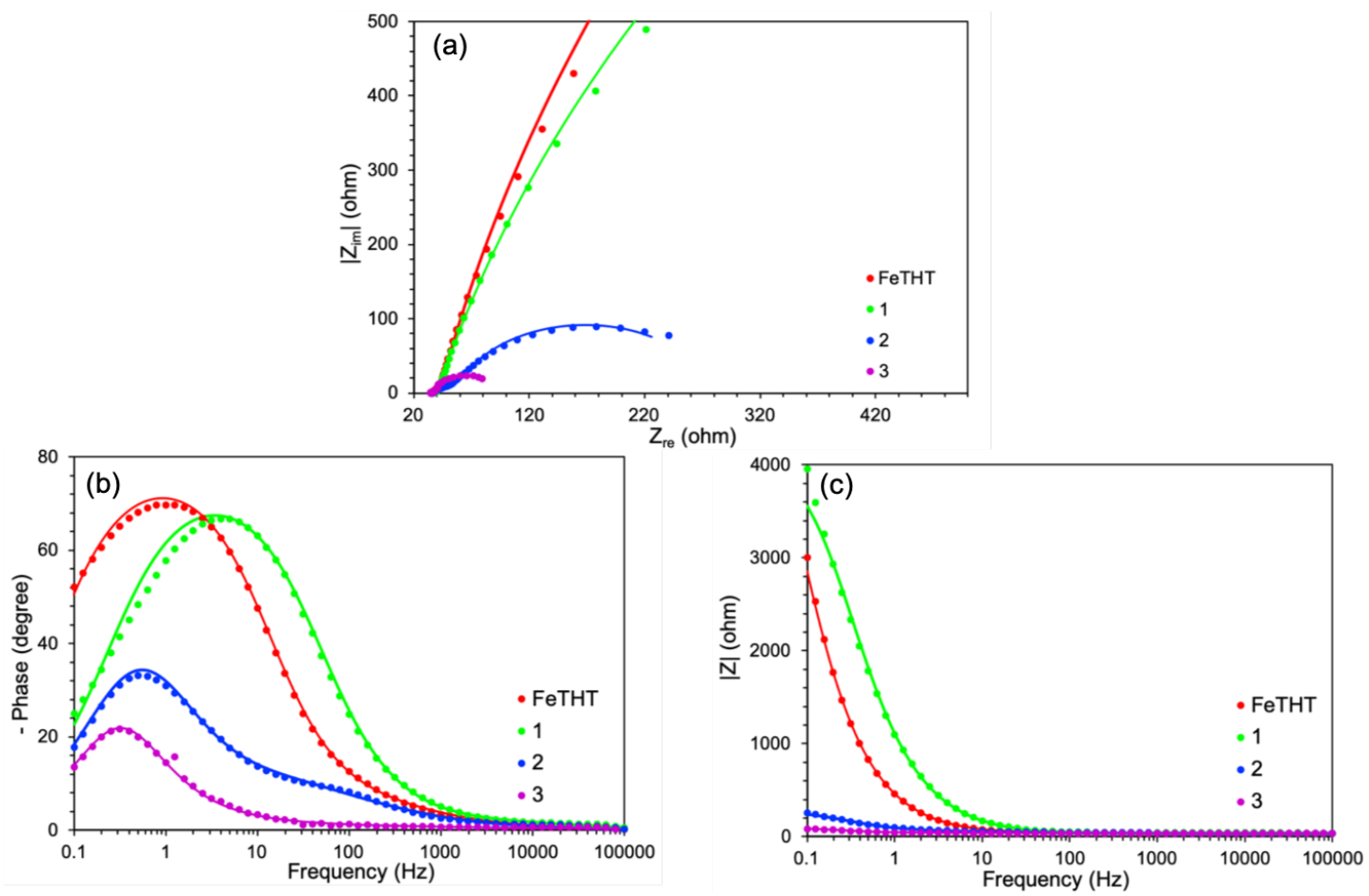

Figure S8. Nyquist (a) and Bode plots (b and c) showing EIS responses of the materials at various overpotentials. For FeTHT and 1, $\eta=368 \mathrm{mV}$; for $\mathbf{2}$ and $\mathbf{3}, \eta=168 \mathrm{mV}$.

Table S4. $\mathrm{R}_{\mathrm{ct}}$ values of the materials derived from fitting EIS data to the 2TS model

\begin{tabular}{|c|c|c|c|c|}
\hline Material & FeTHT & $\mathbf{1}$ & $\mathbf{2}$ & $\mathbf{3}$ \\
\hline$\eta(\mathrm{mV})$ & 368 & 368 & 168 & 168 \\
\hline $\mathrm{R}_{\mathrm{ct}}(\Omega)$ & 5677 & 4190 & 215 & 46.8 \\
\hline $\mathrm{C}_{\mathrm{dl}}(\mathrm{mF})^{a}$ & 0.261 & 1.12 & 2.67 & 15.9 \\
\hline
\end{tabular}

$[a] \mathrm{C}_{\mathrm{dl}}$ is calculated using the following equation: $C_{d l}=\left[\frac{\mathrm{CPE}}{\left(\mathrm{R}_{\mathrm{s}}^{-1}+\mathrm{R}_{\mathrm{ct}}^{-1}\right)^{(1-\mathrm{n})}}\right]^{1 / \mathrm{n}}$ 


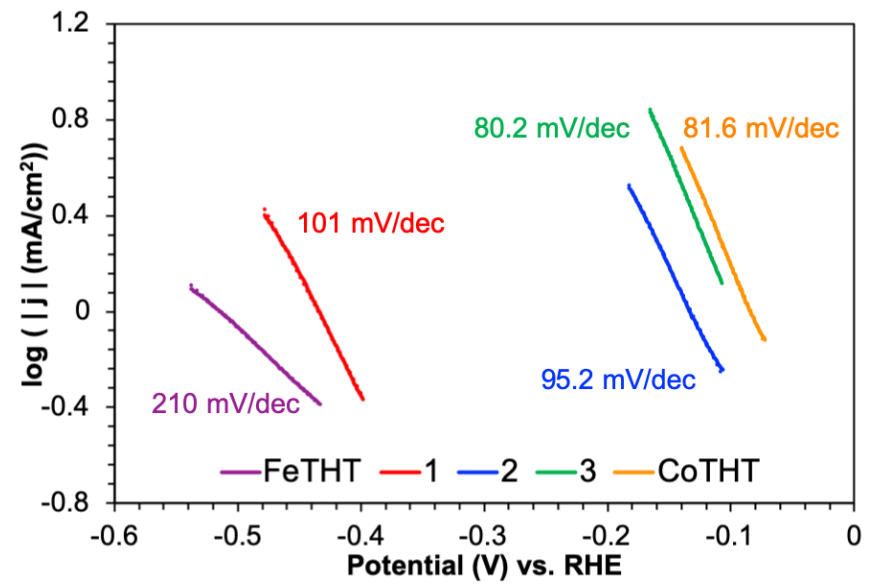

Figure S9. Tafel plots of different materials obtained from polarization curves performed at a scan rate of $5 \mathrm{mV} / \mathrm{s}$ on the $\mathrm{RDE}$ with a rotation rate of $2000 \mathrm{rpm}$.
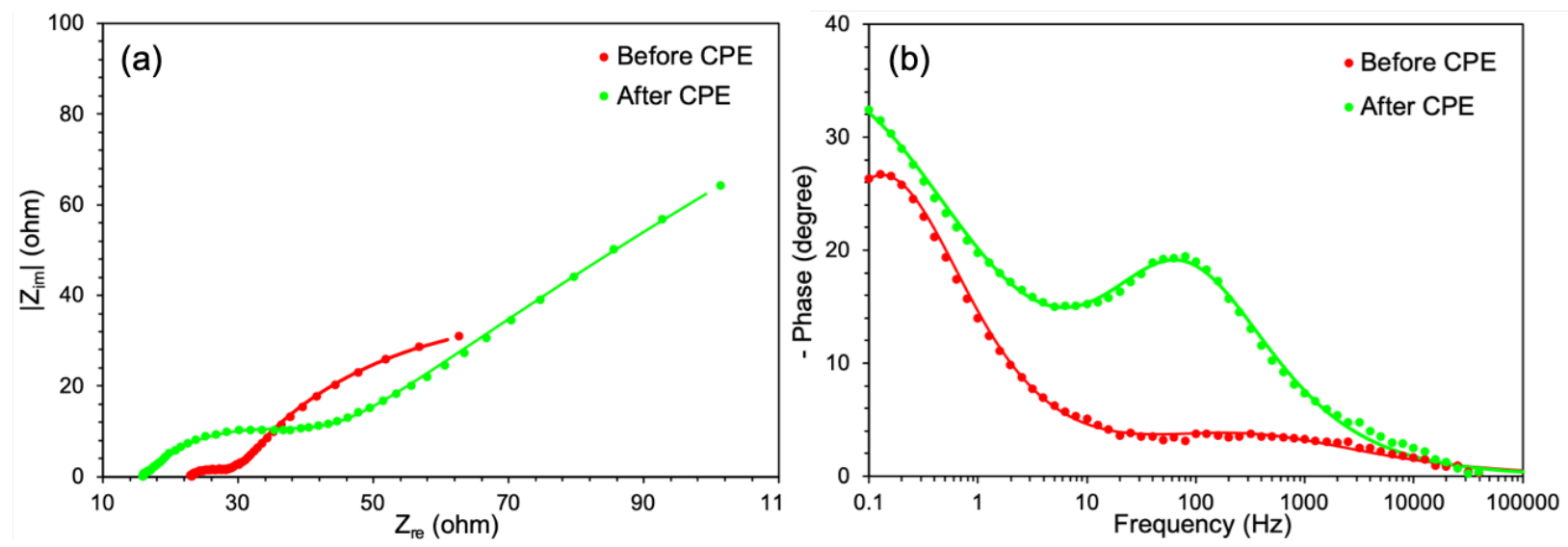

Figure S10. Nyquist (a) and Bode (b) plots showing the EIS responses of 2 at $\eta=168 \mathrm{mV}$ before and after CPE.

Table S5. $\mathrm{R}_{\mathrm{ct}}$ and $\mathrm{C}_{\mathrm{dl}}$ values of 2 before and after CPE, extracted from EIS at $\eta=168 \mathrm{mV}$.

\begin{tabular}{ccc}
\hline Trial & $\mathbf{R}_{\mathbf{c t}}(\mathbf{o h m})$ & $\left.\mathbf{C}_{\mathbf{d l}} \mathbf{( m F}\right)$ \\
\hline Before CPE & 98.5 & 19.3 \\
\hline After CPE & 940 & 4.25 \\
\hline
\end{tabular}


Table S6. Turnover frequency (TON) calculations for 1, 2, and 3 based on the $1 \mathrm{hr}$ CPE experiment shown in Figure 3.

\begin{tabular}{cccc}
\hline MOF & $\mathbf{3}$ & $\mathbf{2}$ & $\mathbf{1}$ \\
\hline Charge Passed (C) & 27.39 & 10.01 & 0.17 \\
\hline $\mathrm{H}_{2}$ Produced (mol) & $1.23 \times 10^{-4}$ & $3.78 \times 10^{-5}$ & $6.25 \times 10^{-7}$ \\
\hline $\mathrm{Fe}(\mathrm{mol})$ & $5.42 \times 10^{-8}$ & $3.64 \times 10^{-7}$ & $1.62 \times 10^{-7}$ \\
\hline $\mathrm{Co}(\mathrm{mol})$ & $9.31 \times 10^{-7}$ & $4.41 \times 10^{-7}$ & $5.31 \times 10^{-8}$ \\
\hline Total metal $(\mathrm{mol})$ & $9.85 \times 10^{-7}$ & $8.04 \times 10^{-7}$ & $2.15 \times 10^{-7}$ \\
\hline $\mathrm{TOF}_{\mathrm{Co}}\left(\mathrm{h}^{-1}\right)$ & 132 & 85.7 & 11.8 \\
\hline $\mathrm{TOF}_{\text {total }}\left(\mathrm{h}^{-1}\right)$ & 125 & 47.0 & 2.91 \\
\hline
\end{tabular}
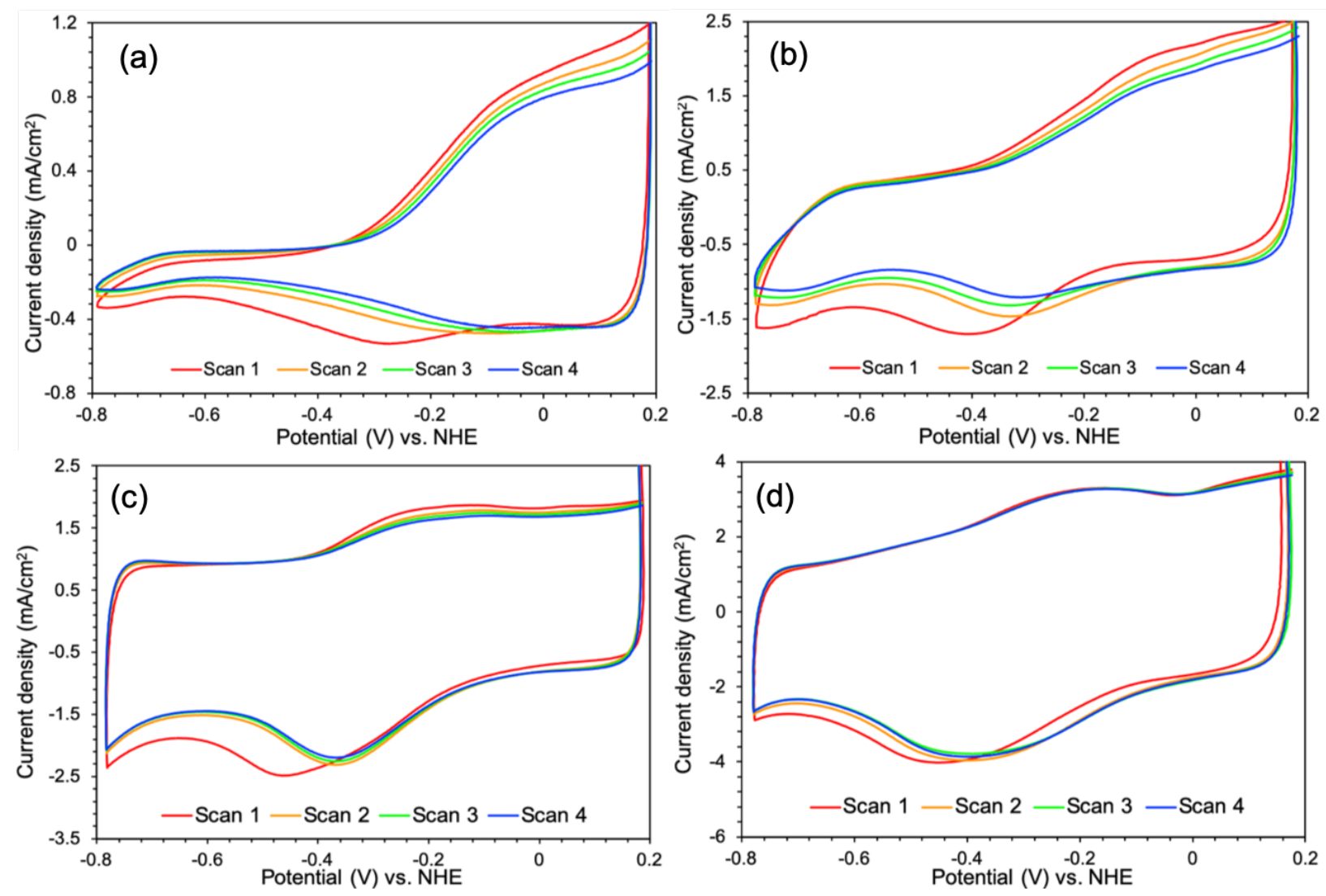

Figure S11. Consecutive CV scans of (a) FeTHT, (b) 1, (c) 2, (d) 3 in $\mathrm{N}_{2}$-saturated $\mathrm{pH} 10$ electrolyte solutions, scan rate: $50 \mathrm{mV} / \mathrm{s}$. 


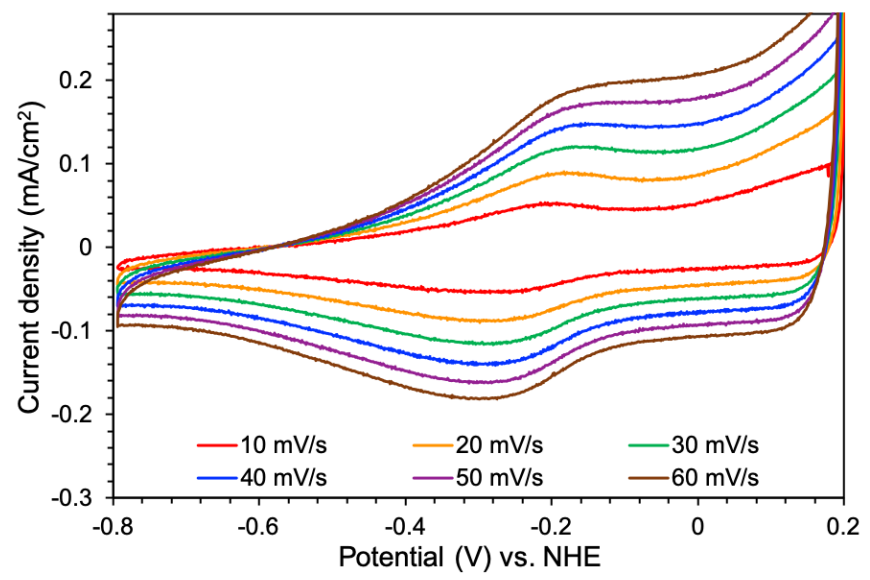

Figure S12. CV scans of CoTHT in $\mathrm{N}_{2}$-saturated pH 10 electrolyte solutions with variable scan rates.
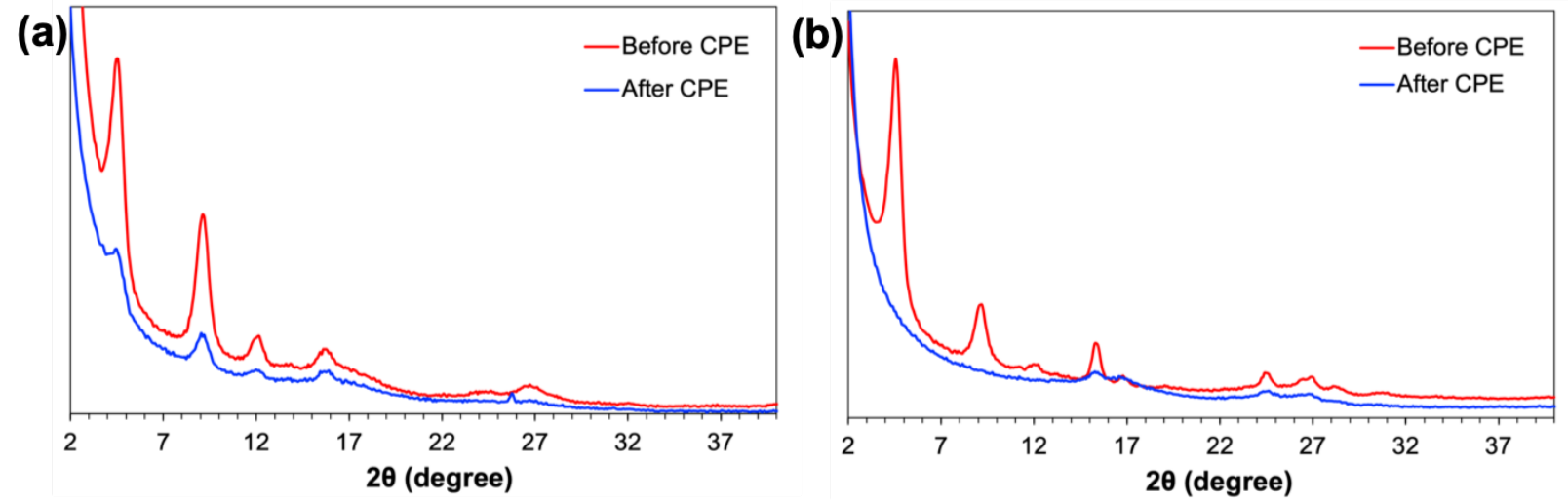

Figure S13. (a) XRD patterns of MOF 2 before and after $1 \mathrm{hr}$ CPE experiment at $-0.418 \mathrm{~V}$ vs. RHE. (b) XRD patterns of FeTHT before and after $1 \mathrm{hr}$ CPE experiment at $-0.518 \mathrm{~V}$ vs. RHE. All measurements were performed in $\mathrm{N}_{2}$-saturated $\mathrm{pH} 1.3$ aqueous electrolyte solutions.
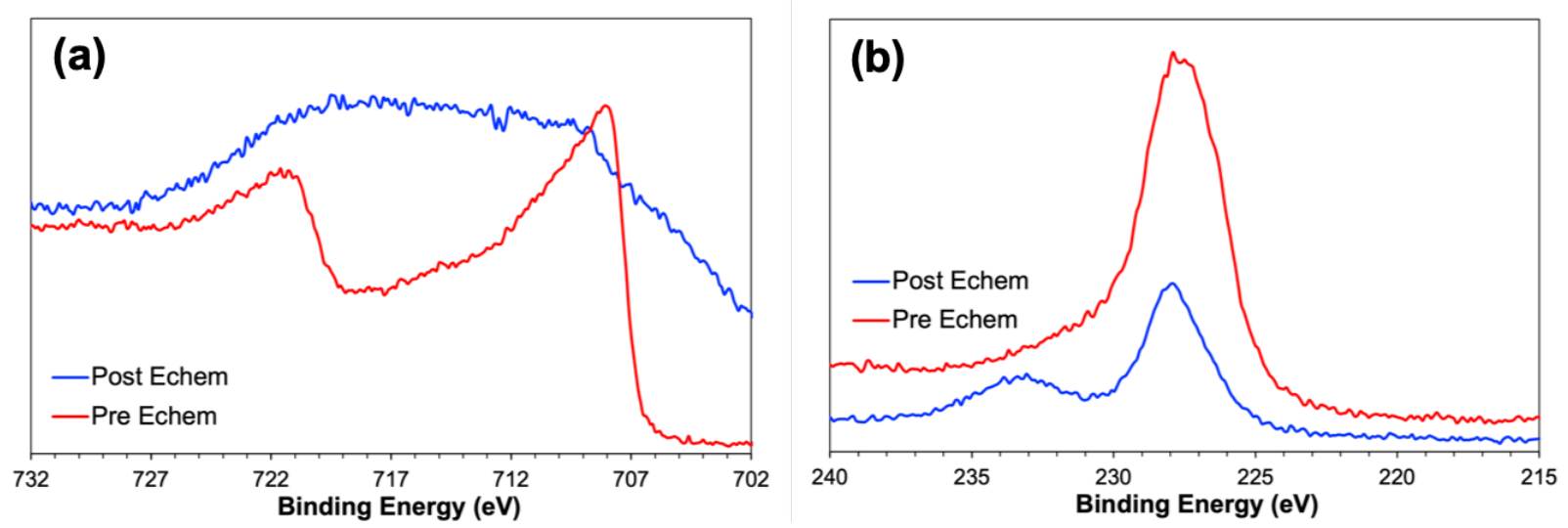

Figure S14. High resolution XPS measurements of FeTHT before and after $1 \mathrm{hr}$ of CPE at $-0.518 \mathrm{~V}$ vs. RHE, (a) Fe 2p region; (b) S 2s region. 

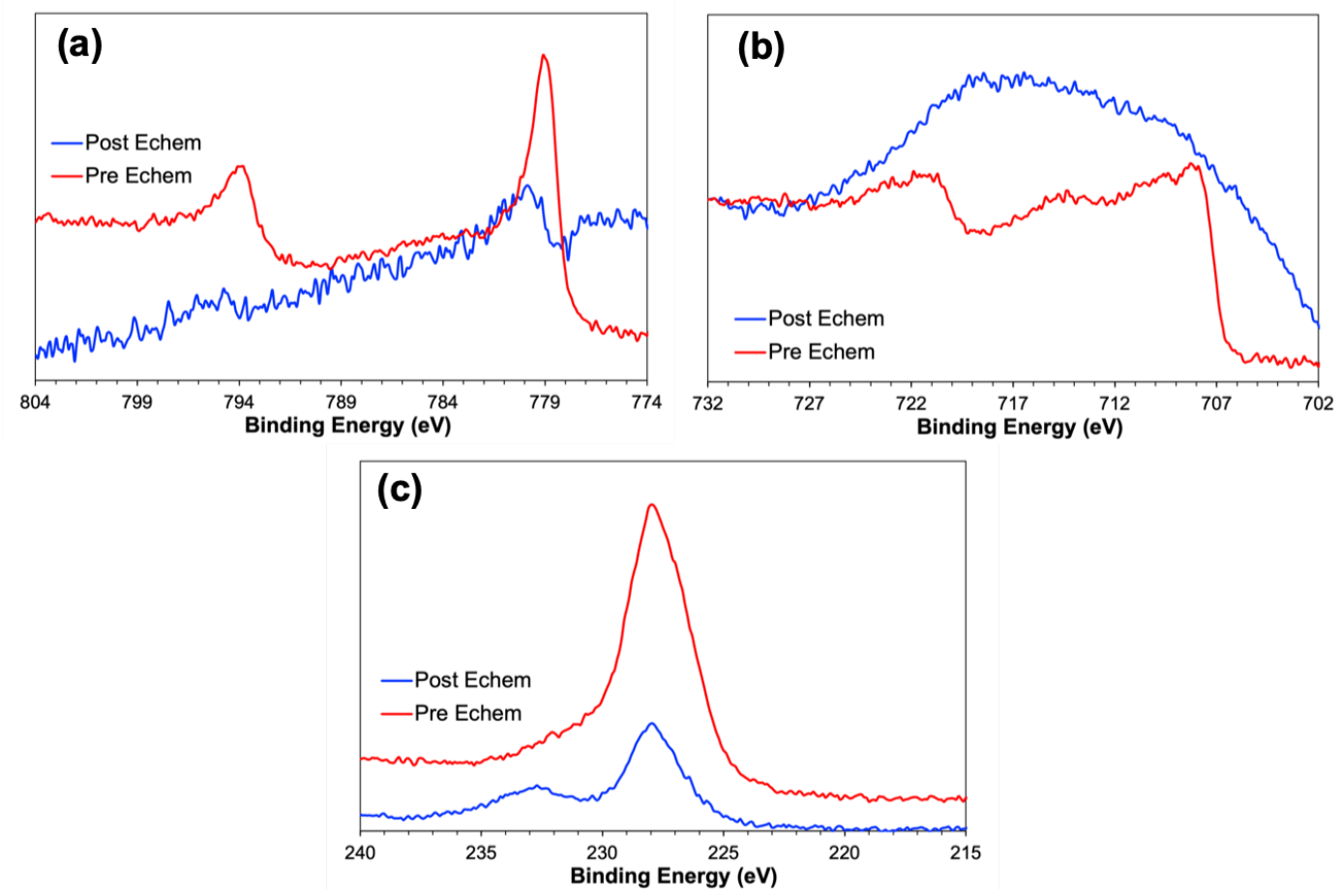

Figure S15. High resolution XPS measurements of MOF 2 before and after $1 \mathrm{hr}$ of CPE at $-0.418 \mathrm{~V}$ vs. RHE, (a) Co 2 p region; (b) Fe 2p region; (c) S 2 s region.

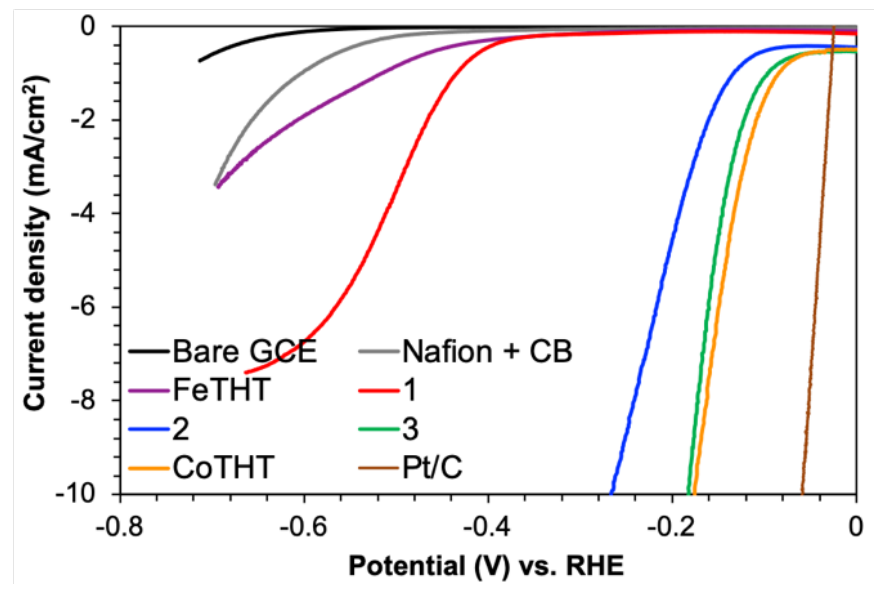

Figure S16. Polarization curves of FeTHT, mixed-metal MOF 1-3, and CoTHT in comparison to the $\mathrm{Pt} / \mathrm{C}$ catalyst. All measurements were performed in $\mathrm{N}_{2}$-saturated $\mathrm{pH} 1.3$ aqueous solutions, scan rate: $5 \mathrm{mV} / \mathrm{s}$, rotation rate: $2000 \mathrm{rpm}$. 
Table S7. Summary of the HER activity of analogous dithiolene-based MOFs.

\begin{tabular}{|c|c|c|c|c|c|}
\hline Catalyst & $\begin{array}{l}\text { Onset } \eta \\
\quad(\mathrm{mV})\end{array}$ & $\begin{array}{c}\eta(\mathrm{mV}) \\
\underset{\mathrm{m}^{2}}{\mathbf{1 0} \mathrm{mA} / \mathrm{c}} \\
\end{array}$ & $\begin{array}{c}\text { Tafel } \\
\text { Slope } \\
\text { (mV/dec) }\end{array}$ & Condition & Ref. \\
\hline FeTHT & 440 & 1 & 210 & \multirow{4}{*}{$\mathrm{pH} 1.3$} & \multirow{4}{*}{$\begin{array}{l}\text { This } \\
\text { work }\end{array}$} \\
\hline CoFeTHT_1 & 400 & 1 & 101 & & \\
\hline CoFeTHT_2 & 117 & 267 & 95.2 & & \\
\hline CoFeTHT_3 & 91 & 183 & 80.2 & & \\
\hline CoTHT (ink) & 61 & 143 & 71 & $\mathrm{pH} 1.3$ & 3 \\
\hline CoTHT (multi-layer) & 403 & 530 & $161^{a}$ & $\mathrm{pH} 1.3$ & 2 \\
\hline CoTHT (single-layer) & 110 & 323 & 82 & $0.5 \mathrm{M} \mathrm{H}_{2} \mathrm{SO}_{4}$ & \multirow{5}{*}{4} \\
\hline CoTHT (powder) & 139 & 496 & 157 & $0.5 \mathrm{M} \mathrm{H}_{2} \mathrm{SO}_{4}$ & \\
\hline CoTHT/graphene & 99 & 426 & 137 & $0.5 \mathrm{M} \mathrm{H}_{2} \mathrm{SO}_{4}$ & \\
\hline $\begin{array}{c}\text { CoTHTA }^{b} \\
\text { (single-layer, } 0.8 \mathrm{~nm} \text { ) }\end{array}$ & 92 & 283 & 71 & $0.5 \mathrm{M} \mathrm{H}_{2} \mathrm{SO}_{4}$ & \\
\hline NiTHTA (single-layer) & 107 & 315 & 76 & $0.5 \mathrm{M} \mathrm{H}_{2} \mathrm{SO}_{4}$ & \\
\hline $\begin{array}{c}\text { NiTHT } \\
\text { (single-layer, } 0.7 \mathrm{~nm} \text { ) }\end{array}$ & 110 & 333 & 80.5 & $0.5 \mathrm{M} \mathrm{H}_{2} \mathrm{SO}_{4}$ & 5 \\
\hline CoBHT (film, 244 nm) & - & 185 & 88 & $\mathrm{pH} 1.3$ & 6 \\
\hline CuBHT (nanoparticle) & 200 & 450 & 95 & $\mathrm{pH} 0.0$ & 7 \\
\hline $\mathrm{NiAT}^{c}$ & 150 & 370 & 128 & $\mathrm{pH} 1.3$ & 8 \\
\hline
\end{tabular}

\section{Computational Methods for FTIR Spectrum Simulation}

The electronic structure calculations were performed using Gaussian 16, Revision C.01 program. ${ }^{9}$ All geometry optimizations and frequency calculations were performed using the B3LYP functiona $1^{10}$ and 6-311G(d,p) basis set. ${ }^{11}$ All geometries were characterized by frequency analysis calculations to be local minima (without any imaginary frequency). As B3LYP tends to overestimate frequencies, a frequency scaling factor of 0.967 was used. ${ }^{12}$ For the IR spectra, the vibrational frequencies were broaden using a IR peak half-width at half height of $20 \mathrm{~cm}^{-1}$.

Due to the large unit cell associated with CoTHT, the calculation of IR spectrum using periodic DFT is prohibitively computationally expensive. Therefore, we created a molecular model of CoTHT with a cobalt atom coordinated by two THT ligands. This geometry was cut from a periodic model of CoTHT that was studied in our previous work. ${ }^{3}$ The sulfur atoms were capped with hydrogen to saturate the sulfur bonds and mimic the periodic system. While the periodic system shows a mixed oxide state of cobalt, for the molecular model, we used a doublet spin state as this gave the lowest energy. The geometry detail is provided below. 


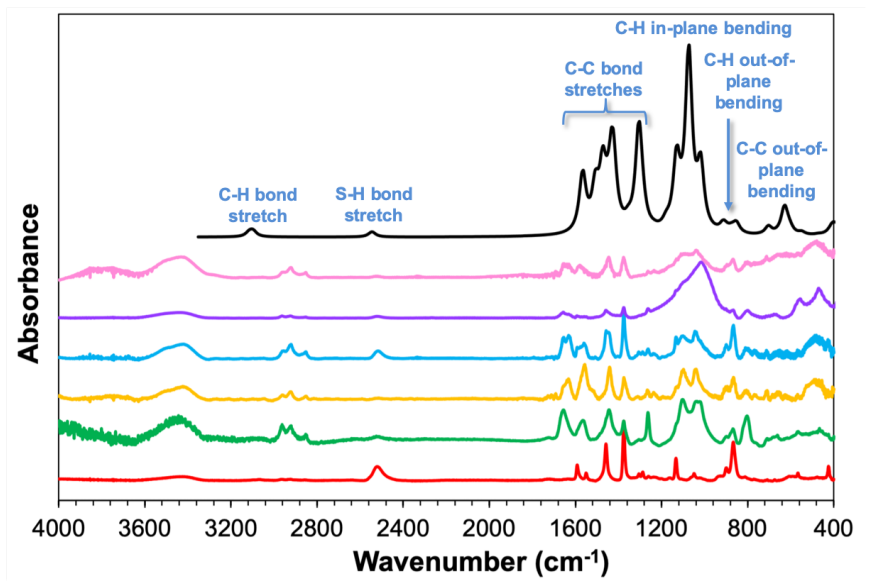

Figure S17. DFT simulated FTIR spectrum of the cobalt dithiolene molecular model (black trace) and the experimental FTIR spectra of the MOF series, from top to bottom: FeTHT (pink); CoFeTHT-1 (purple); CoFeTHT-2 (blue); CoFeTHT-3 (yellow); CoTHT (green); THT ligand (red). The absorption at $3300 \mathrm{~cm}^{-1}$ is attributed to residual water that was introduced during the synthesis.

The Geometry of the Cobalt Dithiolene Molecular Model

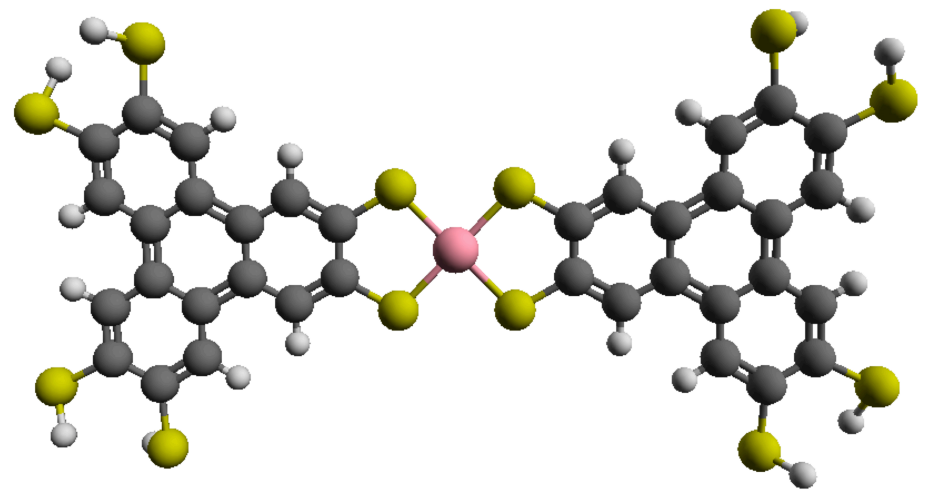
Co $-0.000007 \quad-0.000031 \quad 0.000017$
C $\quad 5.483255 \quad-0.717743 \quad 0.005869$
C $\quad-5.483255 \quad 0.717719 \quad 0.006095$
$\begin{array}{llll}\text { C } & 6.755782 & 1.442618 & -0.011473\end{array}$
C $\quad-6.755807 \quad-1.442628-0.010712$
C $7.985145 \quad-0.734033-0.002647$
C $\quad-7.985138 \quad 0.734044 \quad-0.002262$
C $\quad-5.483265 \quad-0.717746 \quad-0.005719$
$\begin{array}{llll}\text { C } & 5.483248 & 0.717724 & -0.006249\end{array}$
$\begin{array}{llll}\text { C } & -6.755774 & 1.442621 & 0.011171\end{array}$ 

$\begin{array}{llll}\text { C } & 6.755788 & -1.442623 & 0.011003\end{array}$
$\begin{array}{llll}\text { C } & -7.985153 & -0.734018 & 0.002817\end{array}$
$\begin{array}{llll}\text { C } & 7.985137 & 0.734030 & 0.002129\end{array}$
$\begin{array}{llll}\text { C } & 3.037485 & -0.709596 & 0.001471\end{array}$
$\begin{array}{llll}\text { C } & -3.037490 & 0.709561 & 0.001526\end{array}$
$\begin{array}{llll}\text { C } & 7.990728 & 3.567697 & -0.030018\end{array}$
$\begin{array}{llll}\text { C } & -7.990782 & -3.567697 & -0.028737\end{array}$
$\begin{array}{llll}\text { C } & 9.215763 & -2.860260 & -0.034646\end{array}$
$\begin{array}{llll}\text { C } & -9.215697 & 2.860299 & -0.034622\end{array}$
$\begin{array}{llll}\text { C } & -3.037495 & -0.709592 & -0.001297\end{array}$
$\begin{array}{llll}\text { C } & 3.037478 & 0.709559 & -0.001662\end{array}$
$\begin{array}{llll}\text { C } & -7.990681 & 3.567723 & 0.029261\end{array}$
$\begin{array}{llll}\text { C } & 7.990760 & -3.567692 & 0.029428\end{array}$
$\begin{array}{llll}\text { C } & -9.215767 & -2.860237 & 0.035260\end{array}$
$\begin{array}{llll}\text { C } & 9.215730 & 2.860266 & 0.034045\end{array}$
$\begin{array}{llll}\text { C } & 6.801977 & -2.850701 & 0.038714\end{array}$
$\begin{array}{llll}\text { C } & -6.801915 & 2.850703 & 0.038624\end{array}$
$\begin{array}{llll}\text { C } & 4.254451 & 1.390363 & -0.005556\end{array}$
$\begin{array}{llll}\text { C } & -4.254466 & -1.390392 & -0.004963\end{array}$
$\begin{array}{llll}\text { C } & 9.182931 & 1.469522 & 0.039163\end{array}$
$\begin{array}{llll}\text { C } & -9.182953 & -1.469493 & 0.040084\end{array}$
$\begin{array}{llll}\text { C } & -6.801994 & -2.850712 & -0.038187\end{array}$
$\begin{array}{llll}\text { C } & 6.801949 & 2.850695 & -0.039242\end{array}$
$\begin{array}{llll}\text { C } & -4.254459 & 1.390363 & 0.005269\end{array}$
$\begin{array}{llll}\text { C } & 4.254458 & -1.390390 & 0.005277\end{array}$
$\begin{array}{llll}\text { C } & -9.182920 & 1.469550 & -0.039436\end{array}$
$\begin{array}{llll}\text { C } & 9.182942 & -1.469507 & -0.039733\end{array}$
S $7.877533 \quad-5.357165 \quad-0.004860$
$\begin{array}{llll}\text { S } & -7.877392 & 5.357163 & -0.005346\end{array}$
$\begin{array}{llll}\text { S } & 1.521889 & 1.583086 & -0.001255\end{array}$
$\begin{array}{llll}\mathrm{S} & -1.521898 & -1.583128 & -0.000802\end{array}$
$\begin{array}{llll}\text { S } & 10.819999 & 3.635976 & 0.045415\end{array}$ 


$$
\begin{array}{llll}
\text { S } & -10.820041 & -3.635937 & 0.046887 \\
\text { S } & -7.877534 & -5.357128 & 0.005823 \\
\text { S } & 7.877455 & 5.357136 & 0.004173 \\
\text { S } & -1.521889 & 1.583085 & 0.000940 \\
\text { S } & 1.521893 & -1.583134 & 0.001180 \\
\text { S } & -10.819949 & 3.636019 & -0.046191 \\
\text { S } & 10.820027 & -3.635906 & -0.046221 \\
\text { H } & 5.884508 & -3.422363 & 0.091492 \\
\text { H } & -5.884417 & 3.422337 & 0.091284 \\
\text { H } & 4.218394 & 2.472735 & -0.004488 \\
\text { H } & -4.218415 & -2.472763 & -0.003663 \\
\text { H } & 10.132020 & 0.952360 & 0.106394 \\
\text { H } & -10.132033 & -0.952313 & 0.107272 \\
\text { H } & -5.884523 & -3.422373 & -0.090953 \\
\text { H } & 5.884465 & 3.422333 & -0.092065 \\
\text { H } & -4.218414 & 2.472738 & 0.003977 \\
\text { H } & 4.218411 & -2.472763 & 0.004231 \\
\text { H } & -10.132016 & 0.952387 & -0.106534 \\
\text { H } & 10.132017 & -0.952317 & -0.106964 \\
\text { H } & -8.684779 & 5.602733 & 1.052033 \\
\text { H } & -10.428044 & 4.780851 & -0.647890 \\
\text { H } & -10.428290 & -4.780517 & 0.649168 \\
\text { H } & -8.685631 & -5.602662 & -1.051018 \\
\text { H } & 8.684009 & -5.602511 & 1.053271 \\
\text { H } & 10.427853 & -4.781297 & -0.646678 \\
\text { H } & 10.428249 & 4.780712 & 0.647399 \\
\text { H } & 8.685374 & 5.602456 & -1.052855
\end{array}
$$

\section{References:}

(1) Sakamoto, R.; Kambe, T.; Tsukada, S.; Takada, K.; Hoshiko, K.; Kitagawa, Y.; Okumura, M.; Nishihara, H. П-Conjugated Trinuclear Group-9 Metalladithiolenes With a Triphenylene Backbone. Inorg. Chem. 2013, 52 (13), 7411-7416.

(2) Clough, A. J.; Yoo, J. W.; Mecklenburg, M. H.; Marinescu, S. C. Two-Dimensional Metal-Organic Surfaces for Efficient Hydrogen Evolution from Water. J. Am. Chem. Soc. 
2015, 137 (1), 118-121.

(3) Chen, K.; Downes, C. A.; Schneider, E.; Goodpaster, J. D.; Marinescu, S. C. Improving and Understanding the Hydrogen Evolving Activity of a Cobalt Dithiolene Metal-Organic Framework. ACS Appl. Mater. Interfaces 2021, 13 (14), 16384-16395.

(4) Dong, R.; Zheng, Z.; Tranca, D. C.; Zhang, J.; Chandrasekhar, N.; Liu, S.; Zhuang, X.; Seifert, G.; Feng, X. Immobilizing Molecular Metal Dithiolene-Diamine Complexes on 2D Metal-Organic Frameworks for Electrocatalytic $\mathrm{H}_{2}$ Production. Chem. Eur. J. 2017, 23 (10), 2255-2260.

(5) Dong, R.; Pfeffermann, M.; Liang, H.; Zheng, Z.; Zhu, X.; Zhang, J.; Feng, X. Large-Area, Free-Standing, Two-Dimensional Supramolecular Polymer Single-Layer Sheets for Highly Efficient Electrocatalytic Hydrogen Evolution. Angew. Chem. Int. Ed. 2015, 54 (41), 12058-12063.

(6) Downes, C. A.; Clough, A. J.; Chen, K.; Yoo, J. W.; Marinescu, S. C. Evaluation of the $\mathrm{H}_{2}$ Evolving Activity of Benzenehexathiolate Coordination Frameworks and the Effect of Film Thickness on $\mathrm{H}_{2}$ Production. ACS Appl. Mater. Interfaces 2018, 10 (2), 1719-1727.

(7) Huang, X.; Yao, H.; Cui, Y.; Hao, W.; Zhu, J.; Xu, W.; Zhu, D. Conductive Copper Benzenehexathiol Coordination Polymer as a Hydrogen Evolution Catalyst. ACS Appl. Mater. Interfaces 2017, 9 (46), 40752-40759.

(8) Sun, X.; Wu, K.-H.; Sakamoto, R.; Kusamoto, T.; Maeda, H.; Ni, X.; Jiang, W.; Liu, F.; Sasaki, S.; Masunaga, H.; Nishihara, H. Bis(Aminothiolato)Nickel Nanosheet as a Redox Switch for Conductivity and an Electrocatalyst for the Hydrogen Evolution Reaction. Chem. Sci. 2017, 8 (12), 8078-8085.

(9) Frisch, M. J.; Trucks, G. W.; Schlegel, H. B.; Scuseria, G. E.; Robb, M. A.; Cheeseman, J. R.; Scalmani, G.; Barone, V.; Petersson, G. A.; Nakatsuji, H.; Li, X.; Caricato, M.; Marenich, A. V.; Bloino, J.; Janesko, B. G.; Gomperts, R.; Mennucci, B.; Hratchian, H. P.; Ortiz, J. V.; Izmaylov, A. F.; Sonnenberg, J. L.; Williams; Ding, F.; Lipparini, F.; Egidi, F.; Goings, J.; Peng, B.; Petrone, A.; Henderson, T.; Ranasinghe, D.; Zakrzewski, V. G.; Gao, J.; Rega, N.; Zheng, G.; Liang, W.; Hada, M.; Ehara, M.; Toyota, K.; Fukuda, R.; Hasegawa, J.; Ishida, M.; Nakajima, T.; Honda, Y.; Kitao, O.; Nakai, H.; Vreven, T.; Throssell, K.; Montgomery Jr., J. A.; Peralta, J. E.; Ogliaro, F.; Bearpark, M. J.; Heyd, J. J.; Brothers, E. N.; Kudin, K. N.; Staroverov, V. N.; Keith, T. A.; Kobayashi, R.; Normand, J.; Raghavachari, K.; Rendell, A. P.; Burant, J. C.; Iyengar, S. S.; Tomasi, J.; Cossi, M.; Millam, J. M.; Klene, M.; Adamo, C.; Cammi, R.; Ochterski, J. W.; Martin, R. L.; Morokuma, K.; Farkas, O.; Foresman, J. B.; Fox, D. J.: Gaussian 16 Rev. C.01. Wallingford, CT, 2016.

(10) Becke, A. D.: Density-functional thermochemistry. III. The role of exact exchange. The Journal of Chemical Physics 1993, 98, 5648-5652.

(11) Krishnan, R.; Binkley, J. S.; Seeger, R.; Pople, J. A.: Self-consistent molecular orbital methods. XX. A basis set for correlated wave functions. The Journal of Chemical Physics 1980, 72, 650-654.

(12) NIST Computational Chemistry Comparison and Benchmark Database, NIST Standard Reference Database Number 101 Release 21, August 2020, Editor: Russell D. Johnson III http://cccbdb.nist.gov/ 